\title{
INFORMATION MODELING AND LANDSCAPE: INTERVENTION METHODOLOGY FOR READING COMPLEX SYSTEMS
}

\author{
M. G. Cianci ${ }^{*}$, M. Molinari ${ }^{1}$ \\ ${ }^{1}$ Dept. of Architecture, Roma Tre University, L.go G.B. Marzi 10, 00153, Rome, Italy mariagrazia.cianci@uniroma3.it, \\ mat.molinari@outlook.it
}

Commission II

KEY WORDS: Landscape, Survey, Parametrization, Heritage, Point Cloud, BIM, LIM,

\begin{abstract}
:
When we talk about landscape and in particular landscape architecture, we refer to a living structure in continuous evolution and in clear contrast to the aesthetic and geometric immobility of the single architectural building. By landscape we mean a complex set of transformations of a historical and biological nature; the times of these components are very different, but they work in a single cycle and in symbiosis between them. This set is inseparable from what happens in the present, we must always think about who lives the reality and know how to interpret the different subjective perceptions of a space. The theme of landscape representation is a subject of difficult development, not only for the multiplicity of elements involved in a single system, but for the dynamism and continuous transformation of the element that one wants to study. The intrinsic multidisciplinarity in this field imposes different visions on the methodology to be used to understand and in the end represent. We must ask ourselves which is the problem in understanding how nowadays it is possible to develop a system capable of grouping the different needs that arise from the study of the landscape and which tools must be used in surveying and representation. To date, in the Italian context, the landscape project and its graphic representation is steady to the production of static images, realized with two-dimensional or three-dimensional digital drawing software, representing only the frames of reality, within the territory survey, or of future developments regarding projects. This methodology of representation imposes strong limits in the understanding of which systems and elements come into play when one enters into relationship with a living architecture
\end{abstract}

\section{INTRUDUCTION}

Architecture, urban planning, landscape and open spaces design, in past eras, were a different declination of a single discipline. Today they have progressively become independent subjects, not only on a technical level but also from a disciplinary and scientific point of view.

The landscape presents itself as a structuring element of the reality in which we live, a set of agents that forms a complex system, whose perception and the way in which we relate to it is subjective and closely linked to the observer. The ways in which a subject interacts with the anthropic and natural structuring elements of this reality are various, closely linked to the way in which the user interprets the components. (Cianci, 2002). Observing with detachment, participation, interacting and living the territory poses various problems not only related to the way in which the landscape is designed but also how one can represent a continuously moving reality, whose fundamental principle consists in the complete absence of staticity. Focusing on the types of landscape that can be found within our reality, we can identify four main types: mainly natural, predominantly anthropogenic, a mixed landscape and finally an archaeological landscape. Briefly analyzing the typologies listed, one can see how the difficulty in describing them intrinsically reports the resulting subjectivity; for this reason the analysis is mainly focused on understanding the single components that, in a single whole, come to form the complex landscape system. If in architecture we talk about complex projects when we want to describe a multi-level design, including more functions in contexts that are difficult to interpret and design, when we talk about a landscape project we do not use complex landscape definition because the term is intrinsic. As previously mentioned, the union of the anthropic and natural elements generates in itself a complex reality in terms of architectural artefacts and biodiversity.
The research work developed has two main objectives: the first is a work of analysis of the state of the art in the digital representation of the project of open spaces, the second objective concerns the analysis and formulation of a methodology of work and representation integrated of some of the components. The study of the state of affairs is the founding base of the research work, understanding the methodologies through which the project is developed and represented is essential to be able to understand the strengths and criticalities that derive from it. Hence the second objective arises, to understand how the different professions that are involved in a complex project such as open spaces can interact with each other, not only with a view to new transformation but also to maintenance and relief of the existing. Never before today, in 2018, is it fundamental to understand the spontaneous biodiversity of natural landscapes, but also that of our cities. The theme of urban green, of the relationship with the anthropic spaces and the way in which they interact is a current topic that must be tackled, in a scenario in which the territorial consolidation is bringing serious natural imbalances within our realities.

The use of greenery, as a recurrent element not only in new architectures but in new projects of open spaces, is increasingly strong in a view of re-nautralization and sustenance of the cities of the twenty-first century. The research is a first approach to the vision of the design of open spaces as a complex design element that can be developed through the same modus operandi of Building Information Modeling (BIM). In the scope of the study, we want to define the Landscape Information terminology Modeling (LIM), as information systems are applied to the open space and not to the architectural product.

The Italian legislation, but also all the other international regulations are adapting to new technologies, legislating new rules focused on BIM and public procurement, not allowing access to tenders if this modus operandi is not used. The research is part of a field of work still under development and needs to

\footnotetext{
${ }^{*}$ Maria Grazia Cianci
} 
find a working methodology that allows the landscape project to adapt to new technologies and not be stuck inside the classic work patterns. The study of the implementation of these technologies should not be aimed solely at new construction projects but also at the possibility of being able to use them to understand and understand and carry out maintenance work on the urban fabric. (Bianchini, and Inglese 2016)

Many universities on the international scene are working on simplified city and park models through BIM and GIS platforms in order to understand, analyze and predict the development of urban fabric and natural phenomena as opposed to buildings.

In Denmark, municipalities and universities are working in synergy to understand how it is possible to calculate the impact of new buildings, of pollution as opposed to natural elements present in the urban fabric through energy calculation models implemented in the BIM area. (Fig.1)

The possibility of having a digital database of architectural artifacts and natural systems also allows the systematic study and planning of extraordinary and ordinary maintenance interventions. provides for the modeling of a green element and parameterized in such a way as to contain the design parameters that follow along the lines of the botanical card of the plant species.

In this way it is possible to understand its development over time, maintenance interventions such as pruning and protection against viruses and seasonality. (Cianci et al, 2018)

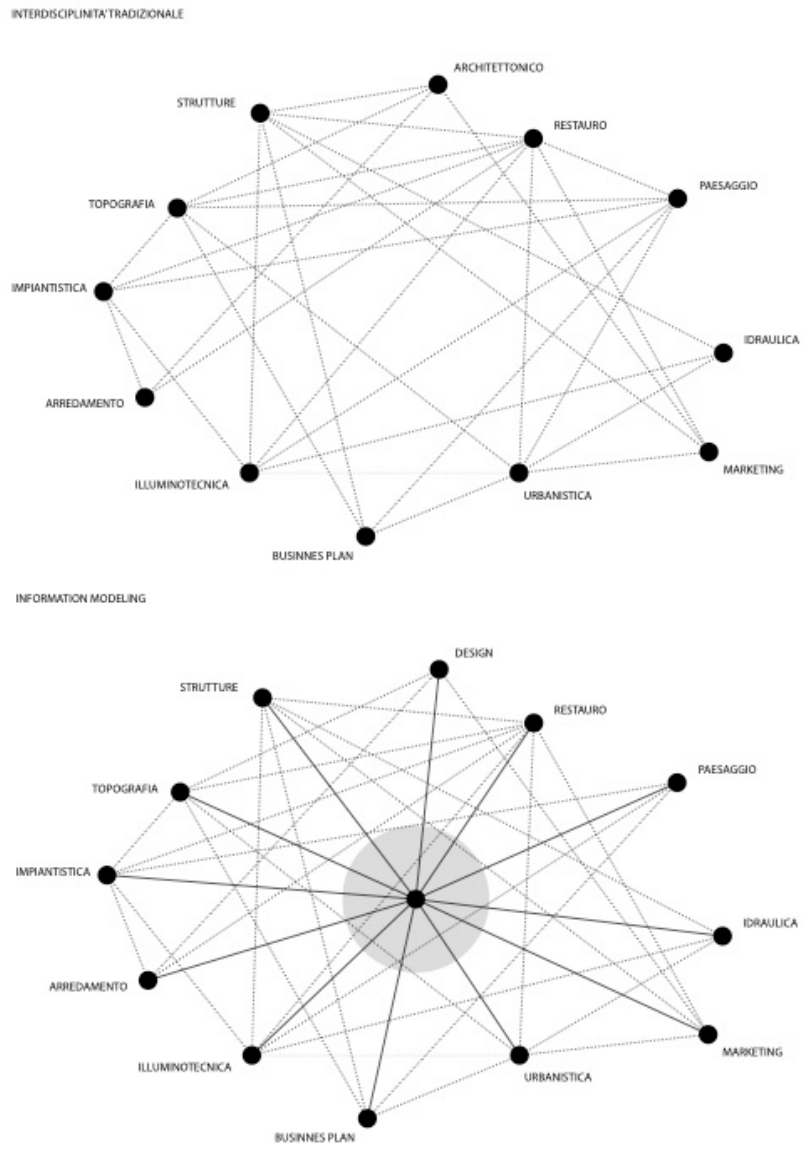

Figure 1: Top to bottom. Diagram of analysis of today's procedural system in the field of design. The disciplines are connected to each other with complex connections but do not interact in a single central model.

Diagram of analysis of today's procedural system in the context of design based on information systems. The disciplines are connected to each other through a single central model.

\section{THE SURVEY OF OPEN SPACES}

"The Drawing is dead, long live the Drawing" as Roberto Maestro intervened at the UID conference on the activity and research of the national group of the ICAR university field ICAR17. With this sentence, Maestro wanted to highlight the difficult condition of the design subject in architectural field. A difficult condition because every technique is constantly evolving, when we consolidate a system of representation that is two-dimensional or three-dimensional, we always tend to overcome it with something new. Over time, the Design of Architecture undergoes a condition of permanent transformation, in correlation with cultural changes and solutions of instruments and executive techniques. the new regulations in the field of public procurement on the Italian and international scene, these methods are still in use but are leaving more and more space for information systems. These consist of a more detailed management of the entire design and construction process within the architectural and landscape project. The information modeling, is becoming a standard on the international scene, affirming more and more on the Italian one, the professionals are leaving the traditional CAD methods to move on to this "new" management of the design process. (Minigucci 2008).

The research through the analysis of this methodology uses traditional techniques as a basis of work and tries to translate the theoretical and practical concepts that apply to the Building Information Modeling to the design of open spaces. To do this, a working methodology was developed based on three steps: surveying, modeling and parameterization. The survey phase is carried out using the modern tools for the recovery of the existing (Laser Scanner, Drone) in order to obtain a 1: 1 scale model of the territory. (Becker et al., 2015). The second phase, the modeling consists of remodeling the natural and anthropic elements detected on BIM oriented platforms, in order to have models that can be parameterized. The third phase consists precisely in being able to assign the parameters and data to the three-dimensional elements. In this case, for the anthropic elements we proceed along the lines of the methodology used in the Building Information Modeling, while for the natural elements we work on a new scale and methodology of representation, translating the concept of LOD to the natural element, but parameterizing it in such a way be able to take into account the growth and decay of the studied element.

The area used as a case study, through which the experimentation of this methodology of work on the landscape has begun, is a portion of the right bank of the Tiber, Lungotevere Testaccio in Rome, the view of the ex-slaughter of the city on the river.

The choice to study this area is due to the complexity of the natural and anthropic structures present. A stratification of elements that leads to the birth of a critical reading on the urban landscape of the city of Rome and its complex systems. Why can we talk about a complex landscape in this case? First of all, for the natural system, a landscape strongly characterized by riparian vegetation, which engages between the beginning of the Muraglioni system whose construction lasted more than half a century ended in 1926, and the area embankment system industrial ex-Gazometer. Secondly, there are architectural stratifications dating back to different eras, generating a vision of the place of great scientific interest. On the river bank taken into consideration there was the ancient route of the Aurelian Walls, specifically, an extension of the posthumous path to the original construction in order to control the floods of the river. Of these traces still remain visible signs, such as: the sighting tower, a stretch of walls and part of the foundations. In close connection with this architectural artifact there is the whole façade of the former slaughterhouse and in the background the ex-gazometro, of the architectures that today can be called industrial archeology. All these elements are involved in giving life to an urban landscape of extreme richness and importance, where the 
knowledge of the orography of the area, the vegetation and the architectural elements becomes fundamental not only for attracting maintenance interventions, but also for the conscious planning actions. The research has developed a specific working methodology to be applied to the BIM computerization of the working model:

- Surveying through tools with output the dense cloud

- $\quad$ Management of the dense cloud (Fig.2)

- Import of the dense cloud in the LIM area

- Modeling of natural and anthropic elements

- Use of the dense cloud in BIM, as a matter of fact

- Assignment of datasets and geometric features to modeled elements

- Management of the LIM model, for maintenance and / or planning.

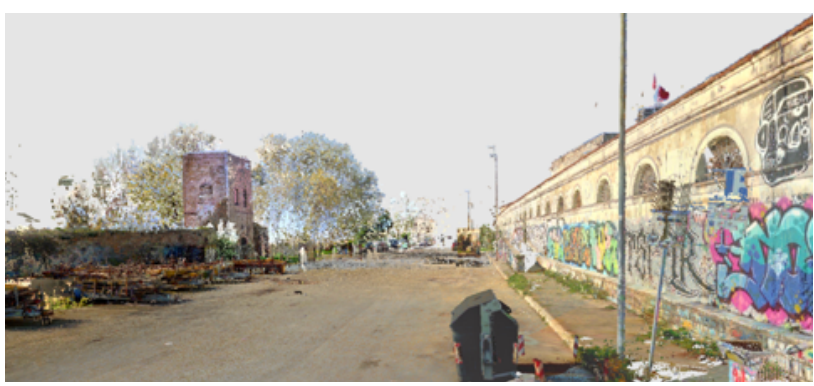

Figure 2: Dense Cloud of the study area, the bank of the Tiber in front of the former slaughterhouse, imported into the Autodesk ReCap management program.

The object of the research is the informative representation of open spaces, focusing the study on some of the territorial, biological components. The first question to be asked in analyzing the complex system of open spaces is how it is possible to detect the existing in order to analyze its geometrical characteristics. The relief of this type of components has always been difficult to develop for two main reasons, the first for the lack of a defined geometry (there are many components present on the territory and each with specific need for transformation) the second for the scale to which you work. Given the difficulty in resuming the geometry of open spaces it was necessary to establish a methodology of intervention for the survey. This methodology, applied to the case study, has the function of a guideline, adaptable to different situations. The need to be adaptable comes from the diversity that derives from every single type of space. Always using as an evaluation tool, the comparison between complex architectural artifact and territory, the motivations for using laser scanners as opposed to traditional relief techniques are many and based on rigorously scientific motivations. An architectural element by its nature has a geometry defined by a general idea of anthropic origin, its forms follow the rules, the knowledge consolidated over time and the schools of thought outlined. All these values, with the exception of exceptions, lead to the coexistence of elements that make it possible to systematize the relevant techniques and processes of the existing. The detection of a historical architecture, using traditional techniques, based on the literature of the sector which shows the canonical geometries of the components of the architectural structure simplifies and facilitates the detection process. The artifacts must be detected and studied as a whole and complex, being well aware of their being a result of a stratification of interventions each performed with the techniques and materials of that period. Therefore, in the architectural field it has become a practice to use point clouds obtained by photomodelling and survey techniques using laser scanners, to speed up and eliminate the percentage of human error present in the normal methodologies. The territory, in particular the natural one, has two components, the anthropic one and the natural one.
These components, present in different percentages according to the type of space analyzed, follow diametrically opposite geometric rules. If for the anthropic elements we can recognize a rule, an intervention methodology, the natural elements precisely because living organisms, or elements molded by atmospheric agents and by the passage of time, present an absence of geometric rules. The survey of an arboreal element, of the course of the terrain, has a possibility and a percentage of error that is much greater than that of the architectural organism. We must also consider the different degree of acceptance of the error between the two types of project. As a concrete beam admits an error in the unit of centimeters and one in iron in millimeters, the relief of the territory being focused on spaces of higher dimensions compared to an architectural artifact is more inclined to accept greater errors. The use of ground-taking technologies through point clouds tends to eliminate this factor almost entirely. The density of the points defined, as mentioned, by the laser beams projected on the surfaces, allows an accurate reconstruction of the natural geometries and not only. (Fig.3)
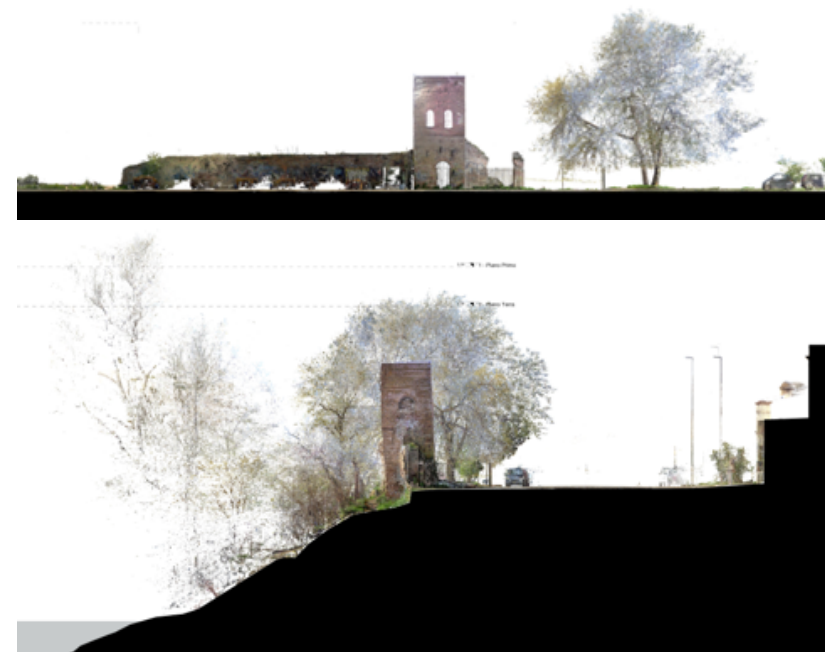

Figure 3: Longitudinal and transversal section, obtained by importing the dense cloud into Autodesk Revit software.

The new frontiers of technology provide ever more precise and complex measuring instruments for the survey of the territory and its monuments. These devices allow to record in a very simple way the geometric and spatial characters of any archaeological or architectural context. However, this apparent instrumental simplification must not mislead us, leading us also to a conceptual simplification of the detection methodologies to be used and of the survey project to be carried out. It is important to underline that for a correct execution of the work there is neither a univocal method nor a universal instrument, but each measurement campaign will consist of a different combination of methodologies and technologies. To give the correct answers to the problems to be studied, the work strategy, therefore, should be established (in the definition phase of the survey project) based on the specific metrics and the instrumental resolution. Obviously at these premises must be added the environmental conditions in which the shooting will take place, the economic resources available, the necessary skills and the tools suitable for carrying out the work. The use of the instruments must not eliminate the procedures implemented up to now in the context of the survey. A good relief is such if upstream there is a preparation of the scanning to be done through the instruments. The survey of open spaces, the orography of the terrain, requires a study that is even more in-depth paradoxically than what one can think of. (Bianchini, 2014) Being in an open space, the instruments do not have a physical limit, but only the arrival length of the laser. Designing a survey campaign with overlapping cloud points of $20 \% / 30 \%$ while avoiding 
unnecessary elements relieves postproduction work and the dense cloud model.

\section{METODHOLOGY OF DIGITSLIZATION}

There are many difficulties encountered in the three-dimensional modeling of the components of open spaces. The volubility of some of these, the different shapes with sinuous and undefined lines as opposed to elements composed of delineated and regular geometries makes this methodology of representation very complex. In the first instance we must understand the reason why, from a design perspective and an open space survey, the elements must be modeled in the three dimensions. Secondly, we need to define what we need to model and why we need to digitize it. GIS systems, as mentioned previously, work for data associations with geometric elements in space: line point and polygon. The metadata connected to these geometries compensate for the lack of geometrical information concerning the element taken into consideration. The obstruction of the object, the altitude, the form, are all defined as text and data strings within the tables connected to the object; through specific scripts you can also insert images, documents or videos to make up for the lack of a three-dimensional vision. This absence of representation, within the following research, can be accepted from the perspective of a vision of a project on a territorial scale, with a purpose aimed at the analysis, planning and maintenance of the territory. Setting a project, a future image of an open space up to architectural elements based only on forms and associations of data impoverishes and flattens the design action. In this context, understanding what and how you want to represent it assumes great importance. As in the architectural project, the three-dimensional model of the architectural apparatus is aimed at the fruition, perception and comprehension of the stylistic choices of the designer also the representation in the three dimensions of the model of an open space falls into these concepts. Before arriving at a precise definition of the elements and methodologies of representation, we must make a premise and understand the purpose for which we arrive at representing the components of the project in a specific way. In the architectural project to date there are two main modeling methodologies, the first that can be defined traditional and consists of a model that highlights specific aspects of the architectural work aimed primarily at the study of composition and final fruition for the client or third parties; the second one, instead, fits within the scope of the Information Modeling work, in the specific case of the buildings the Building Information Modeling (BIM). The reasons why these two models differ are the foundations on which the research is based. A model created within the BIM framework, is aimed at the construction of the architectural project, considering the construction site phases and the existing technological systems, as it eliminates, in the most virtuous cases, the need to graphicize the project, referring everything to the model BIM and the information contained therein. As introduced at the beginning of the paragraph the work in BIM works by degrees of definition to which correspond different geometrical and data attributes; the higher the LOD definition level, the more complex are the geometric components that represent the element and the data it contains. The traditional model, created through NURBS and Mesh design programs, as opposed to the BIM modeling field, is composed of objects far from constructive logic and often are bent by the designer's desire to highlight architectural elements of greater interest by going to in other shadows, coming to a definition of "conscious error". Error as elements existing within the project are not represented, aware as it is the designer to choose not to show them to convey precise concepts. These elements are also included in the strategies for the design of new projects and for the recovery of existing elements of the landscape.

The methodology is based on the use of the dense cloud obtained by laser scanner, or photographic surveying tools such as drones and professional cameras. This resumption of existing elements is not based on the perception of the detector's surroundings but on a non-selective "capture" of all the elements that fall within the range of the instruments used, obtaining a three-dimensional model defined by points in space. The next step, the clean-up, takes into account the singularity of those who redraws what has been detected. The main use that is currently made of the cloud of points of relief is to use it as a basis for two-dimensional redesign of the detected objects, replacing the three-dimensional model of relief with two-dimensional graphic drawings (plan, section). The research wants to pay particular attention to this passage. In the Information Modeling, in presence of an LOD above 200 it is necessary to define a three-dimensional geometry also for the natural elements. In this case the point cloud is used to trace the shapes of natural and anthropic elements not working on the two-dimensional XY Cartesian space but adding the third dimension and the $\mathrm{Z}$ axis.

The design of open spaces, particularly in urban areas, has a higher complexity due to the factors that come into play within the intervention area and on the border areas. The relationship with the fabric of the city elevates the characteristics of the space since there are no longer only natural elements but also anthropic components, very often predominant. The reasoning for the reconstruction of the embankment also applies to these elements. The terrain is no longer just a surface or a three-dimensional object of variable thickness but a real stratification of layers that form the earth element. The reconstruction of the topography is divided into three steps, establish the zero level of the project, in the case under examination is the flat part corresponding to the roadway, draw a surface based on control points, and finally assign to these points, through the commands displacement and alignment, the corresponding points on the dense cloud. In this way it is possible to obtain a surface with an irregular pattern corresponding to the ground detected. You can not work simply through the use of a surface, as the elements that interact with the territory that are natural or anthropic do not have a geometry based on a single xy plane but on the three dimensions. For this reason it is necessary to give a thickness to this element, enriching the topographic element with information. How can this element be enriched? Through pre-existing study documents it was possible to know the trend and the different stratigraphy of the territory. (Fig. 4)

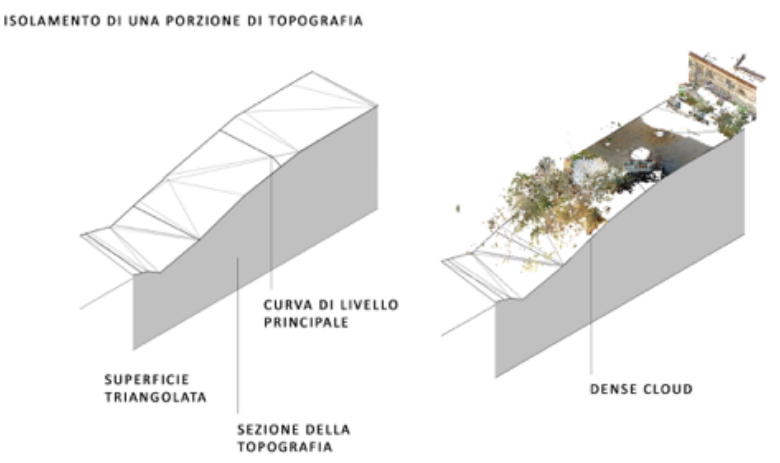

Figure 4: Model developed in the LIM field, within the

Autodesk Revit 2019 software. The topography has been modeled by assigning the elevation points of the surface to the detected points of the dense cloud. In this way the terrain model works as a covering element of the dense cloud, replacing its geometry.

Having this knowledge it is possible to divide the extruded surface, in several layers, by both constant and irregular trends, assigning specific data to each of them. The three-dimensional topographic object obtained, is parametric, and it is therefore 
possible to modify the geometry in the transition from the survey to the project, while retaining the information of the state of affairs in order to have constant control over the changes made. This is possible through the phasing modeling setting, one of the working tools in the information modeling. Perhaps the most complex part is the modeling of trees, defined as articulated because to graphicize and parameterize the trend is in some cases subjective, at the discretion of who is representing the relief and the project. As mentioned before, it is impossible, if not too time consuming within a set workflow, to rebuild the tree element from the point cloud and isolate it. A process that is superfluous in the act of modeling, when the specifics of the single object are so numerous as to make even the reading of the point cloud of the survey difficult. How should we proceed in these cases? The first step after the geometric survey campaign is the survey of the species present within the intervention area; the second one uses the dense cloud as an element to measure the rough bulk of the foliage, the height with respect to the ground and the development of the stem of the element. Given this information it is possible to interact with pre-set tree models and external to the specific project. The intrinsic parameterization within these models is however such as to allow them to be adapted and adapted to each case of work. A fundamental step is to understand how the plant components can be represented in a parametric way both from a geometrical and a data point of view. The cloud, is an element composed of millions of points, with RGB properties depending on the instrumentation used that define the geometric shape of the object detected in three-dimensional space. (Paris 2010) The fundamental concept is precisely the use of points and not of closed lines, solid, surfaces... but of single components, devoid of geometry that give the idea of the element detected. In order to make the shots usable they must be joined, cleaned, post produced and then depending on the use that has to be done, different actions will follow. The research wants to highlight how a well-structured process of surbey and management can allow the use of the dense cloud within a LIM work environment. The dense cloud management process:

- Relief

- Importing the dense cloud into dedicated software

- Cleaning up

- Critical choice of elements

On the market there are many working software for the import and management of the cloud of points each with its characteristics aimed at the type of survey carried out. In the research we chose to use the Autodesk ReCap software in version 2019. The choice of this software unlike others such as Photoscan, the proprietary software of Agiisoft, is dictated by its interoperability with other programs of the Autodesk family such as AutoCad and Revit. Specifically, the desire to work in the LIM field with the point cloud requires a necessary data communication between ReCap and Revit. Importing the point model into dedicated software already makes it possible to start using the dense cloud as an important object without having to go on with more specific actions. In fact, within the software it is possible to measure the detected objects and create orthophoto. It is important to set a clean up process of the dense cloud after exporting it from the survey instruments. The laser scanner detects, as mentioned above, everything that the laser beam can reach, where the beam is interrupted, then the point is found. For this reason, when an open space is detected, elements that are not important for surveying and outside the area of intervention are inserted into the dense cloud. Through a cleaning action you can go to eliminate these cloud points in order to lighten the model and make the relief more focused.

\section{THE PARAMETERS ASSIGNED TO THE COMPONENTS}

Parameterizing an element takes on different meanings depending on the scope of work, this also happens when working in the field of information modeling. There are two main types of parameters, geometric and metadata, and are closely linked but the presence of one is not a necessary condition for the other to be present.

In BIM, an object is defined according to the degrees of accuracy of the representation (the geometrical definition of an element corresponds directly to the data, the LOD). The parameters of the geometry of a component, on the other hand, are specific characteristics that allow a change depending on the insertion of values or the use of modification tools. Parameters can be either specific to a type or a single element. In the architectural field a door with amplitude parameter of the opening defines a value shared by all the doors of that type, while a door with an instance parameter can be part of a typology but different from the others. These same concepts are applicable to all previously exemplified elements.

The concept of dynamism arises from this possibility of integrating, changing, adapting each element on the basis of specific themes. The parameters shared by the elements make a dynamic transformation of the three-dimensional model possible with mathematical formulas, overcoming the static nature of the traditional two-dimensional and three-dimensional drawing. To talk about LIM and dynamism, we need to understand how BIM is such not only for the ability to work interdisciplinary in the three dimensions but because it is possible to overcome them and add others. In the context of the Landscape Information Modeling it is possible to overcome the canonical three dimensions in the same way. The concept of time has already been introduced, a vision in the representation of the project no longer aimed at a distant future years compared to the realization, but based on more temporal arcs. It is important to understand which other dimensions can be transferred from the scope of BIM to the LIM. Costs, management and maintenance and sustainability. All these components joined together in a single work system allow us to talk about dynamism, a digital project that does not stop at the realization of the work but which is in continuous use even in the following years.

Coherently and effectively assigning metadata to natural and anthropic elements is the only way to obtain a dynamic design or model. (Fig.5)

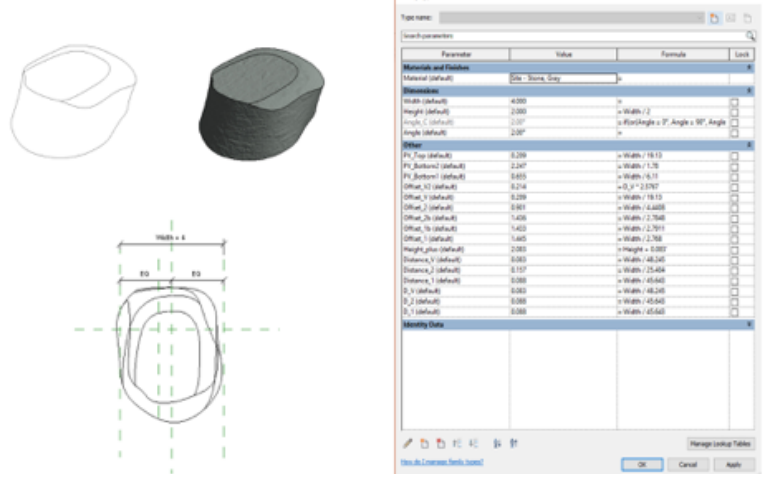

Figure 5: Three-dimensional parametric model of a rocky element. In the image there are: an axonometric textuizzata view, a fil rouge, the plant with one of the constraints and the table with all the priorities. The model is parameterized so as to be able to control the curvature of the edges of the element in addition to the size.

This metadata methodology is a textual design parameter, which makes it possible to insert information in each modeled element aimed at a better understanding and use of the model. The anthropic components contain data related to the material, to the used sub-materials and to cyclical maintenance and replacement periods in case of degradation. For example, a travertine 
threshold, if damaged, already contains data that indicate the way and the part with which it must be replaced. An urban furniture if damaged has an assigned code that corresponds only and only to that type of furniture and therefore avoids problems related to how and with what to replace an element. The natural elements on the other hand show completely different types of metadata and, within the research, work has begun on the transposition and digitalization of the botanical cards within the three-dimensional models. Traditionally, a drawing corresponds to a code which corresponds to a botanical card, which in turn is referred to in the specification sheet. (Marot, 1995) The possibility of obtaining in a single three-dimensional model, the data related to each species allows a greater understanding of each natural element. Already at the design stage, access to a library of three-dimensional elements with all the data relating to geographical location, extension of the foliage, growth, decay, seasonality, flowering, family, allows to implement conscious choices in the identification of species to be used and how to set up a specific planting arrangement. In fact, the sixth of the plant is defined by: sensitivity of the species to intraspefic competition, needs of the species in terms of lighting, breeding system and volume of enclosure of the foliage, soil fertility and water availability; all these are factors that are inserted into the model and can be used for careful planning. (Volk, 2014)

Other fundamental parameters that have been inserted within the family of the natural element are aimed at analyzing the state of health of the plant under survey. The differentiation of these metadata occurs through abbreviations that allow the identification "SD" (Survey Data) and "BD" (Botanic Data). The Botanic Data being objective and always present parameters have been created of type, while Survey Data are instance parameters related to the single element detected. The variable that is more difficult to manage not only in the landscape project, in the open and architectural spaces is time. (Fig. 6) A non-random but certain variable, a factor that in one way or another influences all projects despite using increasingly innovative materials and technologies.
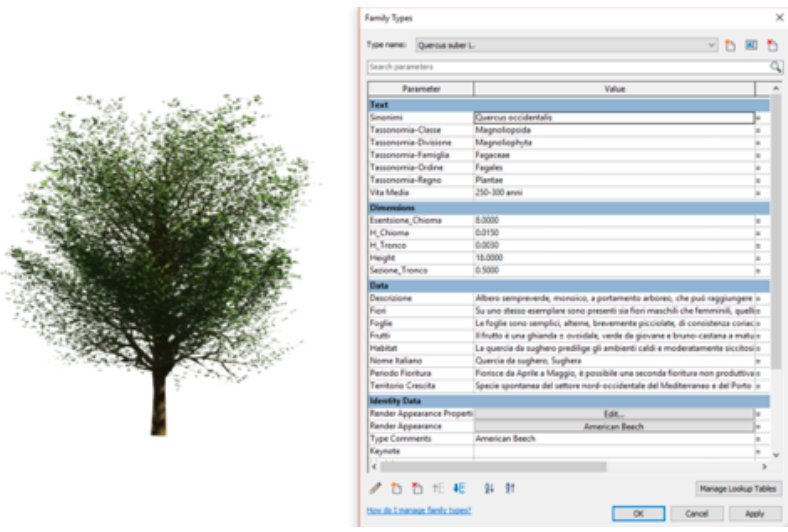

Figure 6: Image taken from BIM software, Autodesk Revit. Through the assignment of parameters it was possible to create a botanical card of type, table on the left. The botanical card of a cork was created in the image

As in structural engineering, the static decays of each element are taken into account to allow a correct physical and mechanical response of the building. It is necessary to behave in the same way with regard to the architectural project and the open spaces. Taking this parameter into account, because in no other way can it be defined, it is very difficult for the way it influences different elements in different ways. An object undergoes a decay of the strength and integrity of the material used over time. How can this variable be inserted into the project? What are the benefits? These are the questions to which the research in progress tries to give an answer in relation to the methodology presented. The difficulty in analyzing time in relation to the design of open spaces flows into having to understand how different elements respond to this variable. The anthropic elements undergo a material and structural decay over time, if not subject to maintenance, the natural elements vary, grow, decay and if not also subject to constant maintenance are damaged. Moreover, the theme of historical analysis of the area under study comes into play, if philological elements of non-existent architectural elements are reconstructed, to recreate an aspect that is not present at present, is not a temporal variable inserted within the project? For this reason, as in the modeling of the individual components that form the open spaces, in this case too, a process of assigning metadata and parameters is carried out to control its development.

Assigning the time parameter can be a difficult concept to understand at first, to understand that everything from a certain point of view can be parameterized is one of the fundamental approaches to transpose the project of open spaces in the BIM.

Two types of temporal parameters must be differentiated, one type is linked to the individual elements that compose the project, a second is inserted directly into the project and allows to check the general progress of the whole project. These two parameters have specific names, the first is a parameter of shared type, with dimensional unit, inserted within the different types of families, the second a global parameter, which is connected through parametric formulas to the shared parameters of the families allows a behavior uniform of design transformations as a function of time. The instrument of time parameterization can have many uses in the representation of the natural elements present in the project of open spaces. This allows us to understand how, according to this parameter, the project green is transformed. The design control proposed here is based on a specific modeling of the families put in place. As explained previously, when one is in the presence of dynamic elements, inclined to change, one of the most suitable parameters to be used is that of visibility associated with a conditional form. This allows us to see an element of height and size, different in relation to how the time period considered changes. The insertion of these parameters together with the concept of visibility allows, in case the life of a natural element ends, to eliminate it from the visualization and to show the situation when this element has lapsed. This tool can be very useful, in correlation with the design phases set in the previous paragraphs to see how the project changes over time.

An example for which this methodology can be applied, are the fifth plants with quincunx with mixed species, when several essences are related, and the growth determines a different aspect of the project in case one essence replaces another. The plant in question taken as an example in fact can be composed of four poplars squared, at a regular distance, with an oak tree in the center. The growth of these two essences is very different, the poplar. (Fig.7)

One of the most innovative aspects developed within the BIM area is the economic dimension of the project, seen no longer as a complementary subject but detached from the development of the project, but as an integral part. The cost is no longer an external element that is calculated in the most virtuous cases at the same time as the definitive and executive development of the project or in the delivery phase to the client, but a datum within the working model, calculable and easy to control. This interoperability of disciplines, estima and design, both architectural or open spaces is possible through the use of the design metadata explained previously. Cost becomes a project data, assigned to each element, based on specific parameters. The cost parameter, like any metadata assigned to the threedimensional model components, can be modified and easily managed using the schedule control tool. These are real work schedules, which if set in the correct way can become real metric calculations. The characteristics described for cost management, which in the architectural field have become a practice (written by an estimator) that through the interdisciplinary work 
environment develops the calculations in symbiosis with the architectural project, are perfectly transferable to the project of space open. The parametric cost control is closely linked, in a correctly set working model, to the work steps chosen at the beginning of the project's LIM re-elaboration. The schedlues allow in fact a perfect interpolation between phases and costs of the project and the drafting of calculation tables according to the design processes. An example is the calculation of the cost of land displacements, calculated on the unit of measurement of the volume. If, in the demolition phase, a soil removal of a certain amount has been set, it is possible to calculate the burdening and transport costs of the removed soil through a mathematical formula of volume / cost relationship.
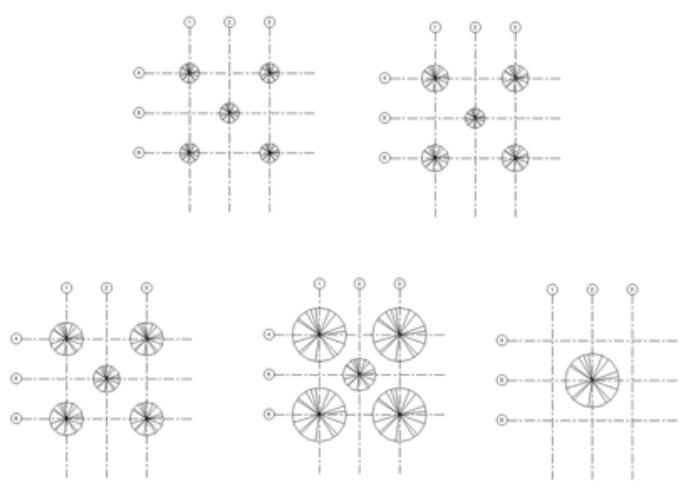

Figur 7. Setting the different phases according to the time parameter on a Populus Alba and Quercus mixed plant system. From left to right: moment of planting, five years after planting, ten years later, fifty years and finally 100 . Through this tool it is possible to immediately see the changes due to the passage of time not only to the scale of detail as in example but also on the scale of the whole project

The same principle is applicable for any other element present in the project area. The green system works in the same way, assigning to the green element two types of cost data, one related to the cost of planting for the project trees and one related to the cost of removal, a parameter that can vary according to age and the type of root system of the element taken into consideration. In fact, through a special conditional tool inside the "if" program, it is possible to assign a type of metadata according to a specific feature of the element connected to another design data.

The ability to understand how costs vary according to design choices can already be seen as a form of project management, with the term managment in English means a real new design dimension. Management of the project means the preparation of a BIM or LIM model able to contain information that allows not only the future development, but also the ordinary and extraordinary maintenance phases. Everything happens as in the case of costs through the use of design metadata and project phases, behaving with two different strategies. The first strategy consists in assigning management metadata in case of damage or replacement of parts of project components through specific global parameters of the project. The second consists in assigning the maintenance or control actions within the descriptive parts in the temporal phases. The first strategy is the most complex in the drafting phase as it requires a capillary and systematic control of the information contained in each element present in the project; despite this, it can be defined as the most complete and least subject to misunderstanding. For example, in the presence of a damaged road surface, a text parameter related to maintenance may contain the necessary information in the use of the same material to avoid points of discontinuity. In the field of natural components the procedure is the same, if elements need replacement, there is a data that defines the process and with what needs to be replaced.

The implementation of management within the project model allows a saving of future costs during the life of the project; despite this, it requires a substantial design investment compared to traditional orders. This is because the writing of the size of the management requires much more information modeling and assignment than a 4 or 5 dimensional model. This happens because with project access to this dimension the design LOD increases with geometric repercussions on all the elements

\section{CONCLUSIONS}

The research presented here, is part of a much broader scope of work aimed at understanding the Landscape not only through its components, but as a complex element, using factors that in this methodology of work are not yet been taken into account. Nevertheless, it is believed that to understand the concept of Landscape, the reading of signs and the relative perception, it is fundamental to proceed with well-defined work steps. Integrating all these concepts within the Information Modeling field, in addition to being very difficult, needs to have solid bases of work before inserting subjective variables within the parametric reading of the landscape project. We have therefore chosen to proceed to the analysis of Open Spaces, and some of the anthropic and natural components that define it. Brian J. Barth, in the February 2016 issue of Landscape Architecure magazine Mgazine writes an article entitled "The Limis of BIM" where he exemplifies his concerns about the future of landscape architecture. In the article Barth highlights how landscape architecture is ready to work in BIM, but that the technologies were not suitable for this aspect of design. The article highlights a fundamental aspect on the British public procurement scene, if landscape architects do not fit in with this new method of representation, they will no longer be able to participate in large public contracts for the creation of new works. In the Italian scene, something is moving, the LAND studio has begun to form a research group aimed at designing a BIM software dedicated to the landscape, leaving (abandoning) the term BIM and using only LIM. (Naixiao 2014)

The research is part of these themes, with the desire to find a solution, not through the use of other proprietary programs, but using the most common software used on today's architectural scene. A constantly evolving research, which breaks down the landscape project into different elements, aims to provide critical and technological tools to the field of design and representation. The decision to start this research by analyzing the representation of some of the components of the Open Space project using a urban context within the city of Rome as a case study has welldefined motivations. Integrating a new work methodology, in a consolidated representation process, is difficult, integrating this new methodology in a context where there is a lack of instruments, moreover not suitable, it is even more so. Working in a context of urban open space, with a high percentage of anthropic elements compared to natural ones, allows greater control over the methodology and tools to be used. To restrict the initial analysis of natural components, differentiating vertical and horizontal elements and going to select only a few, allows us to begin to identify specific situations and to solidify the theoretical knowledge of those elements. The working methodology as explained in the text has been divided into the following steps:

- Surveying through tools with output the dense cloud

- Management of the dense cloud

- Import of the dense cloud in the LIM area

- Modeling of natural and anthropic elements

- Using the dense cloud in BIM, as a matter of fact

- Assignment of datasets and geometric features to modeled elements 
- Management of the LIM model, for maintenance and / or planning

Each of the hypothesized work steps, it is believed, leads to seeing the representation and design of the space in a different way compared to traditional techniques. The dense cloud, today in many contexts used incorrectly, when elaborated with the ultimate aim of representing only a state of fact, is reinterpreted in its use. It is no longer just an object for the visualization and acquisition of two-dimensional documents, but a base on which to set up a three-dimensional critical modeling work by importing in the LIM area. (Bohms 2008)

Using geometries based on geometric parameters and variable data to reconstruct the elements of the open space of both relief and design led to the conception of dynamism. There is talk of dynamism given the ability to change the representation of each component through common project data. The implications of this concept are numerous and still under investigation, but the one that is considered most interesting is the temporal variable of a space. The time parameter has allowed, within the research, to examine how through single commands, it is no longer necessary to redesign the geometry to represent the project in the different time phases.

\section{REFERENCES}

Becker S., Michal P., Dieter F., 2015. Grammar- Supported 3d Indoor Reconstruction From Point Clouds For "As-Built” Bim, In ISPRS Annals of the Photogrammetry, Remote Sensing and Spatial Information Sciences, Vol. II-3/ W4, 2015, pp. 17-24

Bianchini C., 2014. Survey, modeling, interpretation as multidisciplinary components of a Knowledge System, In SCIRES- IT-SCIentific RESearch and Information Technology, 4/1, pp. 15-24.

Bianchini C., Inglese C., Ippolito A., 2016. The role of BIM (Building Information Modeling) for representation and managing of built and historic artifacts, DISEGNARE CON vol.9 No. 16

Bohms, M., 2008. The Building Information Modelling (BIM) Landscape: Facts and Opinions- Action and Results.

Cianci M.G. 2002. La dimensione figurativa del paesaggio - la rappresentazione del paesaggio nel progetto contemporaneo in: Il disegno dell'architettura tra tradizione e innovazione, Gangemi Editore, Roma 2002

Cianci M.G., Calisi D., Molinari M. 2018. Il BIM come strumento di controllo: Recupero delle aree di stazionamento bus e dei locali passeggeri nei nodi di scambio COTRAL di Ponte Mammolo a Roma, in Dn Building Information Modeling, Data \& Semantics, a. I, n.2, dei - Tipografia del Genio Civile

Marot, S. (1995) 'The landscape as alternative', in: K. Vandermarliere (ed.) Het Landschap / The Landscape. Four International Landscape Designers. Antwerp, De Singel, pp 9-36

Minigucci R., 2008. La comunicazione del progetto nell'era digitale. In DISEGNARECON 1(1), pp. 5-19

Naixiao S., 2013. Landscape design based on computer aided design technology. Lecture notes in electrical engineering, Inform and Manage Sci. 257-262

Paris. L. 2010. Quantità e qualità nell'utilizzo dello scanner laser 3D per il rilievo dell'architettura, in X Congreso International Espresiòn gràfica aplicada a la edificaciòn. Alicante, 2, 3 e 4 dicembre 2010, Marfil , Alcoy, vol. I, p. 279-289

Volk, R., Stengel, J., Schultmann F. 2014. Building Information Models (BIM) for existing buildings - literature review and future needs - Automation in Construction 38, pp.109-127 